\title{
Thoracoscopic lung resection for lung cancers in right upper lobe with anomalous structures (displaced B2 and Uvpbi): Report of two cases
}

\author{
Hiroaki Kuroda ${ }^{* 1}$, Mingyon Mun ${ }^{2}$, Ken Nakagawa ${ }^{2}$, Sakae Okumura ${ }^{2}$, Yukinori Sakao ${ }^{1}$ \\ ${ }^{1}$ Department of Thoracic surgery, Aichi Cancer Center Hospital, Kanokoden, Chikusa-ku, Nagoya, Japan \\ ${ }^{2}$ Department of Thoracic Surgical Oncology, Japanease Foundation for Cancer Research, Cancer Institute Hospital, Ariake, \\ kotouku, Tokyo, Japan
}

Received: July 1, 2015

Accepted: September 20, 2015 Online Published: October 8, 2015

DOI: $10.5430 /$ css.v2n1p37

URL: http://dx.doi.org/10.5430/css.v2n1p37

\begin{abstract}
We present two patients with lung adenocarcinomas in the right upper S2 (posterior segment) with anomalous structures (a displaced B2 and the upper lobe vein posterior to the bronchus intermedius), who underwent thoracoscopic right posterior-segment segmentectomy (case 1) and thoracoscopic right upper lobectomy (case 2). Sufficient preoperative examination can significantly aid thoracoscopic surgery (TS). To the best of our knowledge, this is the first report of a TS together with an anomalous vein and displaced bronchus.
\end{abstract}

Key Words: Anomaly, Thoracoscopic segmentectomy, Video-assisted thoracoscopic surgery, Segmentectomy, Displaced B2, Right upper lobe vein posterior to the bronchus intermedius

\section{INTRODUCTION}

Bronchus abnormalities and the pulmonary vein anomalies are relatively rare, and the coexistence of such anomalous structures in a single clinical case is even less common. However, with the spread of thoracoscopic surgery (TS) for lung cancer in recent years, it has become important for surgeons to understand the area where the divergence of the bronchus forms and also how the pulmonary artery is distributed before performing a pulmonary operation. On occasions when such operations are performed, sufficient preoperative understanding of the exact intrapulmonary structures enables surgeons to evade unnecessary bleeding, thus ensuring a safe operation. In our two cases, preoperative identification of the individuals' bronchus and vascular configuration is useful for ensuring safe and infallible TS even if the anatomy differs from the normal.

In this report, we reported two cases with anomalous structures found in the right upper lobe during TS. Preoperative identification of these anomalies was performed using multidetector row computed tomography (MDCT), which is a safe and accurate method. Here we describe in detail our procedures and schemes for TS segmentectomy and lobectomy in the upper lobe with anatomical structures.

\section{CASE PRESEntation}

\subsection{Case 1}

A 61-year-old man underwent annual CT screening. Grandglass opacity (GGO) was observed in the right upper lobe on

\footnotetext{
*Correspondence: Hiroaki Kuroda; Email: h-kuroda@aichi-cc.jp; Address: Department of Thoracic surgery, Aichi Cancer Center Hospital, 1-1 Kanokoden, Chikusa-ku, Nagoya 464-8681, Japan.
} 
MDCT (see Figure 1a). The preoperative sagittal view from the MDCT showed the following characteristics: (a) the maximum size of the object was $10 \mathrm{~mm}$ for the lung window and $0 \mathrm{~mm}$ for the mediastinal window (slice width $=1.25 \mathrm{~mm}$ ); (b) the right upper lobe vein posterior to the bronchus intermedius (UVPBI) flowed into the inferior pulmonary vein (see Figure 1a, b); (c) translocation of B2 from the bronchus intermedius was also present (see Figure 1b); (d) the dorsal major fissure was incomplete, and both "basal segment and S6" and "posterior segment (S2) and S3" demonstrated signs of hypersegmentation. We confirmed the displaced B2 using a bronchofiber scope (see Figure 1c) and depicted the branching patterns of the pulmonary artery and vein (see Figure 1a). A right S2 segmentectomy was performed.
Our intraoperative advisory notes and work scheme are as follows (see Figure 1d-f): (1) V2 (the branch of the UVPBI) and A2 were detected from the dorsal side and interlobar fissure; (2) forceps were transferred from the peripheral side of the displaced B2 to the dorsal side while observing the UVPBI on the monitor, enabling us to distinguish between V6 and V2; (3) the UVPBI was preserved because it was sufficiently away from the tumor; (4) the exposure of V2a (S1 and S2) and V2c (S2 and S3) and the identification of the hypersegmentation ( $\mathrm{S} 2$ and $\mathrm{S} 3$ ) enabled us to form the S2 plane, enabling the identification of the inflated-deflated (ID) line. The final pathological diagnosis was minimally invasive adenocarcinoma.

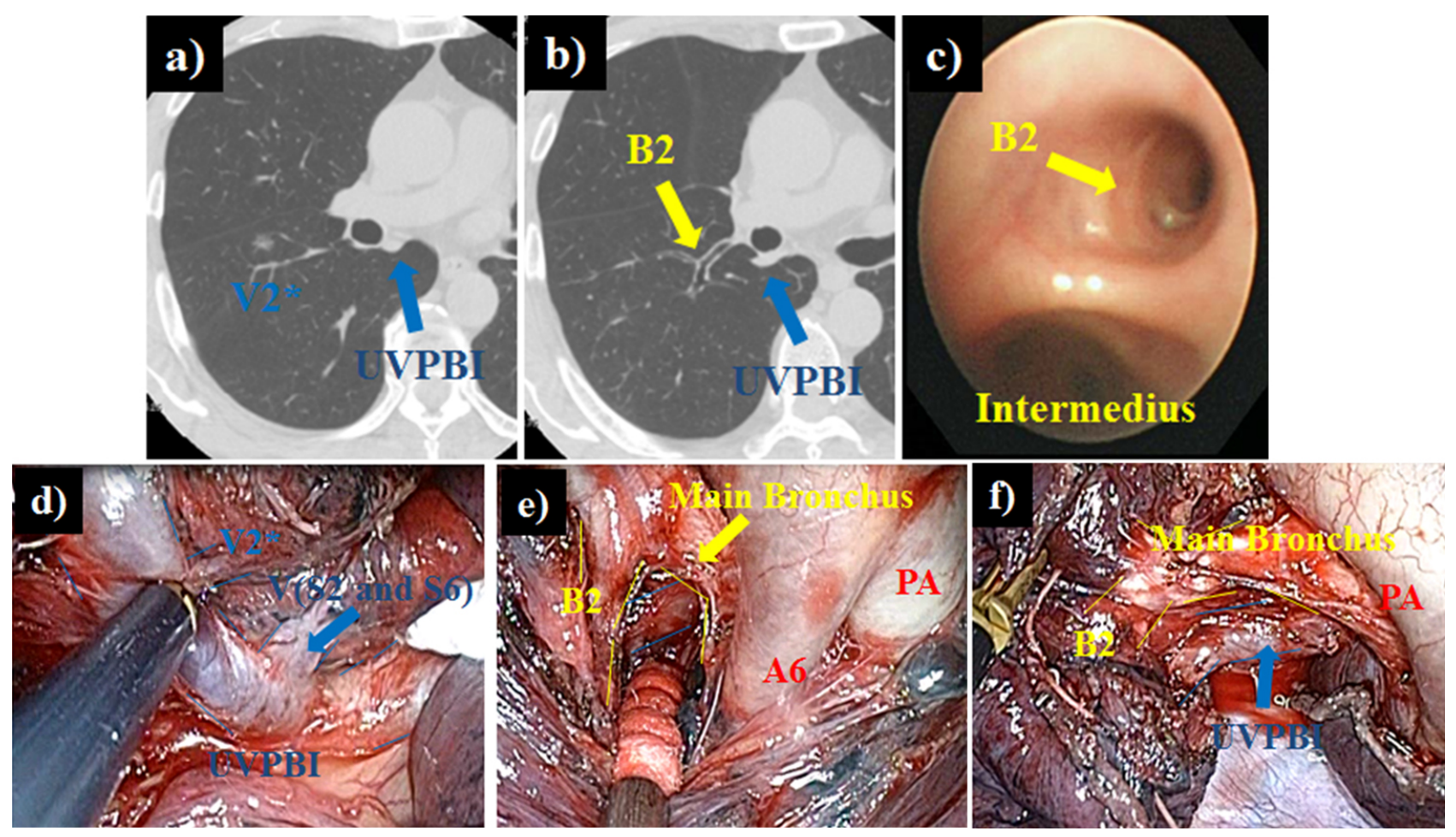

Figure 1. Thoracoscopic right $\mathrm{S} 2$ segmentectomy

a) Chest CT showing ground-glass opacity in the right S2 and the UVPBI running posterior to the bronchus intermedius (blue arrow); b) Chest CT showing the displaced B2 (yellow arrow) and UVPBI (blue arrow); c) Bronchoscopic finding of the displaced B2 diverging from the bronchus intermedius (yellow arrow); d) Intraoperative dorsal finding showing the inter-lobe vein (S2 and S6) (blue arrow) and V2* from the UVPBI. The UVPBI flows into the inferior pulmonary vein; e) Intraoperative inter-lobe finding showing the position of the $U V P B I$ through the peripheral side of the displaced $B 2$ to the dorsal side; f) Intraoperative inter-lobe finding showing the displaced B2 and $U V P B I$.

\subsection{Case 2}

A 54-year-old man was referred to our institution because of an abnormal shadow observed on a chest CT. The preoperative sagittal view on MDCT was as follows: (a) the maximum size was $27.6 \mathrm{~mm}$ for the lung window and $4.3 \mathrm{~mm}$ for the mediastinal window (slice width $=1.00 \mathrm{~mm}$ ) (see Figure 2a); (b) the UVPBI directly flowed into the left atrium as the segmental vein (see Figure 2b); (c) translocation of $\mathrm{B} 2$ from the bronchus intermedius was also present (see Figure 2c). We confirmed the displaced B2 by only 3D-CT reconstruction (see Figure 2c). A right upper lobectomy and lymphadenectomy was performed. Our intraoperative advisory notes and work scheme are as follows (see Figure $2 \mathrm{~d}, \mathrm{e}$ ): (1) forceps were transferred from the peripheral side of the 
displaced B2 to the dorsal side while observing the UVPBI into the left atrium. The final pathological diagnosis was on monitor, enabling the formation of the major fissure; an invasive adenocarcinoma of a predominantly papillary (2) the UVPBI was removed surgically within the forma- nature. tion of the dorsal major fissure because of its direct flow

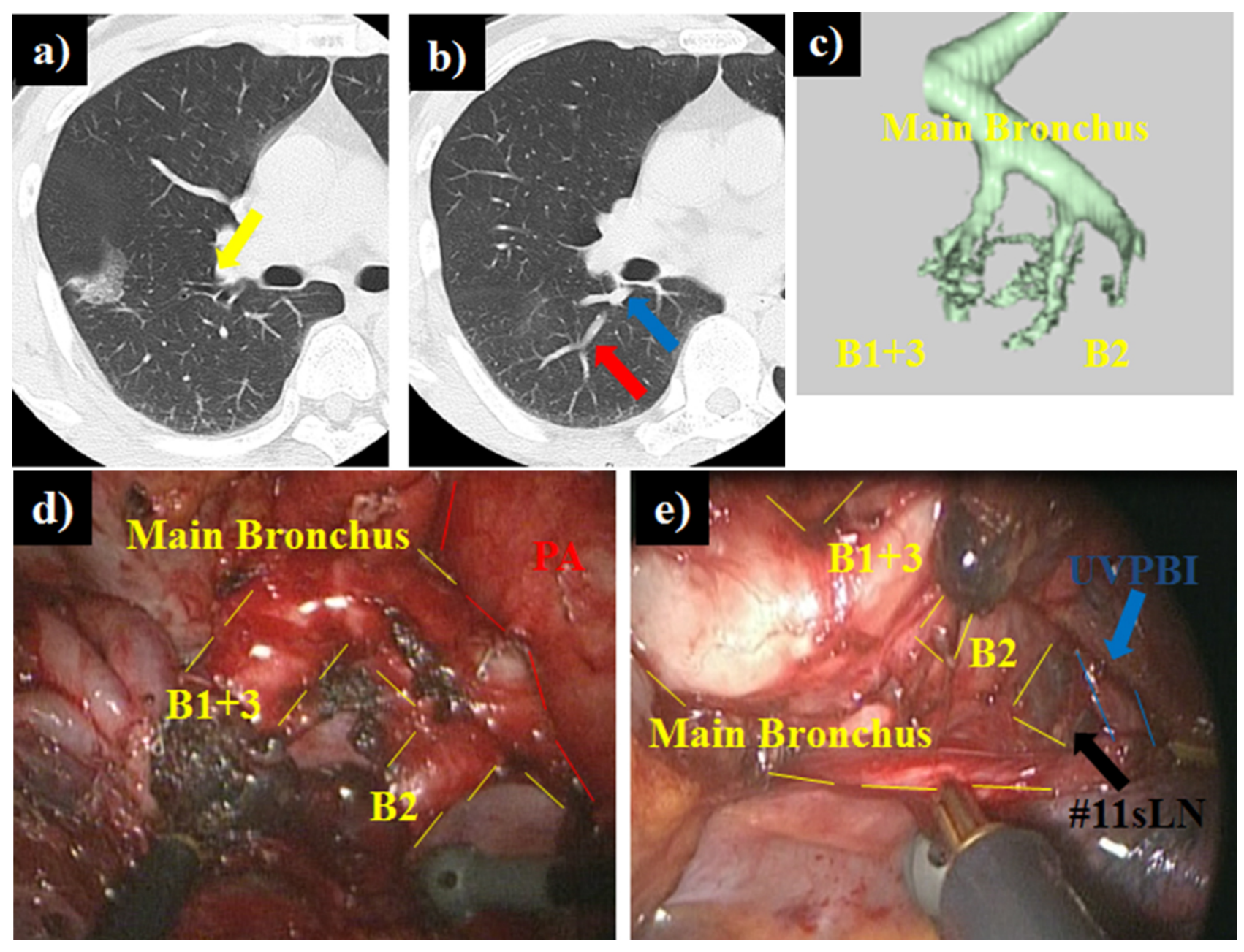

Figure 2. Thoracoscopic right upper lobectomy

a) Chest CT showing A2 (red arrow) and the UVPBI running posterior to the bronchus intermedius (blue arrow); b) Chest CT showing ground-glass opacity, a core in the right $S 2$, and the displaced B2 (yellow arrow); c) 3D schema showing the displaced B2 diverging from the bronchus intermedius; $d$ ) Intraoperative inter-lobe finding showing $B 1+3$ and $B 2$ together, which is compatible with the preoperative $3 D$ schema; $e$ ) Intraoperative dorsal finding showing $B 1+3$ and $B 2$ together and the UVPBI running along the peripheral side of the inter-lobe (\#11s) lymph node.

\section{Discussion}

Some authors have encountered venous variations and recommended both a perioperative and operative evaluation of the vessels. ${ }^{[1,2]}$ Among these variations, the UVPBI is not a rare case. Asai et al. reported that variations in the UVPBI were found in $41(5.7 \%)$ of $725 \mathrm{CT}$ cases and in $9(3.9 \%)$ of 230 right thoracotomy cases. ${ }^{[3]}$ Three venous drainage sites were observed: (a) the superior pulmonary vein group, $55 \%$; (b) the inferior pulmonary vein group, $41 \%$; and (c) the superior segmental vein group, $4 \%$. In this case, the anomalous Published by Sciedu Press

Published by Sciedu Press vein is equivalent to (b) in case 1 and (c) in case 2.

Displacement of the bronchus represents about $75 \%$ of bronchus-related abnormalities. ${ }^{[4]}$ Among them, three bronchus abnormalities are well-known: (a) the right upper lobe related anomalies, $70 \%$; (b) B1 +2 branches from the left main bronchus, 20\%; and (c) B6a branches from the intermediates, $10 \%$. Feofilov et al. reported that approximately $10 \%$ of individuals demonstrate discrete forms of proximal or distal displacement of segmental or subsegmental bronchi in the same lobe, which are easily recognized by 
CT. ${ }^{[5]}$ In our two cases, B2 was found to bifurcate from the proximal side of the intermediate bronchus, and the $\mathrm{S} 2$ was ventilated by the displaced bronchus, which remained normal. The Japanese series based on the Foster-Carter classification reported that the frequency of bronchial abnormalities was $0.3 \%$ to $0.68 \%$, with significant divergence abnormalities focusing around the right upper lobe in particular $(63 \%$ to $80 \%) .{ }^{[4,6]}$ Ohta et al. reported that the frequency of B1 +3 originating from the right main bronchus first was $13.6 \%$ in the displaced segmental bronchus. ${ }^{[4]}$

In our experience, preoperative MDCT evaluation to identify anomalies is useful before performing TS. We were able to detect the pulmonary vessels without medium contrast. However, when 3D-CT is used, a more detailed understanding can be achieved. We considered that the most probable TScausing UVPBI injury is the formation of the posterior aspect of the major fissure. In case 1, Segmental veins (V2 and V6) can be easily approached from the dorsal side of the upper lobe. However, the lung tissue of S2 has to be resected from the interlobar fissure because it is located within the lateral portion of the right upper lobe and the identification of the incomplete lobar fissure. In our novel technique of TS, we approached the UVPBI from the interlobar fissure, followed by tunneling along the V2 peripheral side of the vessel wall and segmental veins to the interlobar fissure, enabling the combined resection of S6 and S2 to be both simple and safe. In contrast, in case 2, we approached the UVPBI from the interlobar fissure, followed by tunneling along V2 through the central side of the vessel wall using the inter-lobe lymph node (\#11LN) as a waypoint, enabling the combined resection of V2 and formation of the major fissure. In addition, the use of new technology, such as using an iPad to report the 3D-CT during TS, might help to more precisely perform an anatomical segmentectomy, particularly in unusual cases.

In conclusion, anomalies of the pulmonary vein, artery, and bronchus are not rare. Preoperative consideration of the axial view on the MDCT and complete analysis of the sagittal and coronal views provides enough information on the pulmonary vessels and bronchus. Moreover, anomalous findings are not an exception. Therefore, sufficient preoperative investigation of the procedure and discussion with surgeons concerning unexpected events can help accomplish complete TS. To the best of our knowledge, this is the first report of a TS with the UVPBI and a displaced B2.

\section{REFERENCES}

[1] Akiba T, Marushima H, Takagi M, et al. Preoperative evaluation of a tracheal bronchus by three-dimensional 64-row multidetector-row computed tomography (MDCT) bronchography and angiography: report of a case. Surg Today. 2008; 38: 841-843. PMid: 18751951. http://dx.doi.org/10.1007/s00595-007-3717-z

[2] Oizumi H, Endoh M, Takeda S, et al. Anatomical Lung Segmentectomy Simulated by Computed Tomographic Angiography. Ann Thorac Surg. 2010; 90: 1382-1383. PMid: 20868860. http://dx.d oi.org/10.1016/j.athoracsur.2009.11.062

[3] Asai K, Urabe N, Yajima K, et al. Right upper lobe venous drainage posterior to the bronchus intermedius: preoperative identification by computed tomography. Ann Thorac Surg. 2005; 79: 1866-1871. PMid: 15919274.http://dx.doi.org/10.1016/j.athoracsu r.2004.12.014

[4] Ohta S, Saito Y, Ueda K, et al. Tracheobronchial anomalies: report of 71 cases. Journal of Japan Society for Bronchology. 1986; 8: 122-130.

[5] Feofilov GL, Osipov VP, Bushuev AA. Anomalies of bronchial deviation. Grudn Khir. 1978; 6: 49-54. PMid: 720941.

[6] Foster-Cater AF. Broncho-Pulmonary Anomalies. Br J Tuberc. 1946; 40: 11-124. 